\title{
Investigation of Distracted Pedestrians' Exposure Duration at Signalized and Unsignalized Pedestrian Crossings: A Bayesian Modeling Approach
}

\author{
Vasiliki-Maria Perra ${ }^{1 *}$, Socrates Basbas ${ }^{1}$, Andreas Nikiforiadis' ${ }^{1}$, Nikolaos-Marios Militsis \\ ${ }^{1}$ School of Rural and Surveying Engineering, Faculty of Engineering, Aristotle University of Thessaloniki, AUTh Campus, \\ 54124 Thessaloniki, Greece \\ * Corresponding author, e-mail: vmperra@certh.gr
}

Received: 25 March 2020, Accepted: 24 May 2020, Published online: 28 February 2022

\begin{abstract}
During the last decades, distraction caused by mobile phones has created concerns about pedestrians' safety, especially while crossing a road. The aim of this paper is to investigate the factors that have an effect on the crossing duration of pedestrians, distracted by mobile phone use, at both signalized and unsignalized crossings. Pedestrians with mobile phones were observed during crossing the aforementioned types of crossings in Thessaloniki, Greece, while their crossing duration was further examined via the development of Bayesian regression models, one for unsignalized and one for signalized crossings. For the research purposes, 554 pedestrians were observed at unsignalized crossings and 409 at signalized ones. The most commonly observed type of distraction was talking on the mobile phone, while texting was also found to be very common. The variables, found to be associated with crossing duration, were significantly different between unsignalized and signalized crossings. Crossing duration at unsignalized crossings seemed to be affected by the experience of a potential conflict with a motorized vehicle and the presence of additional distractors, such as the carrying of an object and the presence of company. At signalized crossings, duration tended to be influenced by the age of the pedestrian and the position before crossing. Current research contributes in understanding and modeling distracted pedestrians' behavior when crossing streets.
\end{abstract}

Keywords

distracted walking, mobile phone, pedestrian behavior, exposure time, Bayesian modeling

\section{Introduction}

Mobile phone use while driving has been proven distracting and has been associated with severe and fatal road accidents. Although the effect of mobile phone use on drivers has been studied thoroughly and relative countermeasures have already been developed and implemented, the relevant research on distracted pedestrians' behavior remains limited, although the surveys that have been carried out so far indicate that mobile phone can be equally distractive in the case of the pedestrians. More specifically, according to recent findings, the significant increase in mobile phone ownership and use during everyday activities, can also lead to fatal incidents during walking and road crossing (Gary et al., 2018).

An important parameter related to pedestrians' safety is crossing duration, since it reflects the duration of exposure to potential risks related to crashes with vehicles. Further research on the exposure duration of pedestrians while crossing is needed, in order to understand the way they behave and the factors that drive their behavior. Identifying these factors can firstly facilitate the development of valid simulation models for pedestrians' behavior and secondly can lead to the implementation of appropriate policies and countermeasures towards pedestrians' safety. These interventions may include the installation of countdown signal timers that inform pedestrians on the remaining time for adapting their walking speed and safely crossing the road (Lambrianidou et al., 2013; Paschalidis et al., 2016), the installation of painted stencil markings or LED signals near or on crosswalks (Barin et al., 2018; Sobhani and Farooq, 2018), the conduction of campaigns, the enforcement of regulations etc.

Taking into account the above, the current paper emphasizes on the crossing duration of pedestrians, attempts to investigate the behavioral characteristics of pedestrians, 
who are being distracted by the use of a mobile phone in various ways, both at signalized and unsignalized crossings. More specifically, it is sought to identify factors that have a significant role to play exclusively on distracted pedestrians' exposure time. With this aim, an observation survey has been carried out, taking into consideration a wide range of crossing behavior parameters before and during the crossing of both signalized and unsignalized crossings and a variety of additional crossing distractors. The research concludes with the development of two models (one for unsignalized and one for signalized crosswalks), which aim to identify and quantify the impact of various factors on crossing duration and consequently to contribute in adding knowledge about how distracted pedestrians behave.

\section{Literature review}

Distraction caused by mobile phone use can be divided into four types:

1. cognitive;

2. visual;

3. auditory and

4. physical.

All these types of distraction have a negative impact on user's ability to perform attention-demanding tasks and effectively analyze the road circumstances (World Health Organization and NHTSA 2011). These types of distraction are regarded as common for both drivers and pedestrians (Hatfield and Murphy, 2007; Pizzamiglio et al., 2017), while further investigation concerning their effects on pedestrians' crossing behavior is necessary for understanding and mitigating distracted walking and eliminating the likelihood of their involvement in traffic accidents.

Taking into consideration research findings so far, the type and level of distraction depends on the performed phone task. For instance, talking on the phone is considered to be a cognitively-demanding task that can affect situational and environmental awareness (Hatfield and Murphy, 2007; Hyman et al., 2010; Neider et al., 2010; Tapiro et al., 2016). Texting, gaming or using apps can reduce visual attention as the user's attention is mainly engaged on the phone screen rather than on environmental surroundings (Chen and Pai, 2018; Chen et al., 2017; 2018; Feld and Plummer, 2019; Haga et al., 2015; Jiang et al., 2018; Krasovsky et al., 2017; Lin and Huang, 2017; Magnani et al., 2017; Tapiro et al., 2016), increasing traffic lights violation and the probability of a collision with a vehicle. The use of headphones can cause limited auditory attention by covering environmental noise (Barton et al., 2016; Chen and Pai, 2018; Magnani et al., 2017; Wells et al., 2018), which is an important stimulus that facilitates safe road crossing.

Recent studies are focused on the physical distraction caused by mobile phone use, showing that it can affect gait performance, by leading to gait patterns' variability, walking speed reduction, as well as walking instability (Agostini et al., 2015; Jiang et al., 2018; Lamberg and Muratori, 2012; Licence et al., 2015; Lin and Huang, 2017; Magnani et al., 2017; Pizzamiglio et al., 2017; Plummer et al., 2015). The findings that emphasize on the reduction of walking speed, are very important in the case of road crossing, as the increase of time needed by a pedestrian for crossing expresses an increased exposure time to risks, such as a collision with a motorized vehicle.

Pedestrians' crossing behavior while using a mobile phone has been investigated mainly through naturalistic, experimental and simulation observational surveys (Lennon et al., 2016; Mwakalonge et al., 2015; Scopatz and Zhou, 2016). Naturalistic observational surveys implement a more holistic approach, having thus the advantage of being carried out under realistic circumstances on realworld settings. Thus, the collection of information about all crossing-related risks and distractors and the optimization of the quality of the collected data are facilitated.

The research findings of naturalistic observational surveys vary, depending on whether the crossing site is signalized or unsignalized. According to Chen et al. (2018) and Russo et al. (2018), road crossing with a mobile phone at signalized intersections can result in traffic lights violations. On the other hand, research focused on unsignalized intersections showed slower road crossings and less checks on traffic before and during the crossing (Hatfield and Murphy, 2007; Mohd Syazwan et al., 2017; Pešić et al., 2016). Taking also into account that the majority of unsafe road crossings and accidents involving vulnerable road users are usually observed at unsignalized intersections (Galanis et al., 2017; Muley et al., 2017), distracted pedestrians' behavior at both signalized and unsignalized intersections needs to be further explored, conducting comparative analysis.

Also, a limited number of studies attempt to develop statistical models for distracted walking in order to investigate in depth the associations between performed mobile phone 
tasks and pedestrians' crossing behavior and generalize and predict the phenomenon. Previous researches set the:

1. traffic lights violation (Russo et al., 2018),

2. mobile phone tasks performed (Ortiz et al., 2017; Russo et al., 2018),

3. safe crossing (Pešić et al., 2016; Sobhani and Farooq, 2018), as dependent variables.

There are also limited studies, which are dealing with distracted pedestrians' crossing speed or duration, which seem to be affected based on how the pedestrian was using the mobile phone. For instance, according to the study of Russo et al. (2018), pedestrians that were talking through the phone or texting, did not change their walking speed, while those using headphones crossed the road faster. On the contrary, Ropaka et al. (2020) observed that mobile phone use, including texting and web-surfing, had a negative impact on crossing speed, especially in the cases when the pedestrian was older and/or accompanied with high pedestrian volumes crossing the road.

The crossing speed and the crossing duration have been also examined in different contexts, not necessarily for distracted pedestrians, but also in the cases of pedestrians who share common characteristics (e.g. children). Based on literature review, crossing speed is affected by the intersection control type, with the relevant results varying between signalized and unsignalized intersections and crossings through midblocks. More specifically, Hassouna (2020) observed that pedestrians cross the unsignalized intersections faster than the signalized ones, while Zafri et al. (2019) noticed slower crossing speeds in corresponding cases. Likewise, crossing was associated with gender, age, company group size, pedestrian flow and volume, the carrying of objects and in some cases with the use of a mobile phone. In particular, Kadali and Vedagiri (2019) observed that male pedestrians presented more changes of their crossing speed and Zafri et al. (2019) that female pedestrians crossed the road slower than males. Regarding the age, several studies found out that young people between 16 and 30 years old were more likely to change their crossing speed (Kadali and Vedagiri, 2019), presenting higher crossing speeds (Forde and Daniel, 2020) in comparison to children, elderly and disabled people (Bansal et al., 2019; Zafri et al., 2019), who crossed slower.

Concerning company, crossing speeds were slowing down as the size of the company group was growing (Bansal et al., 2019; Kadali and Vedagiri, 2019;
Shaaban, 2019). The same effect was noticed when pedestrians were crossing while holding objects, such as bags or luggage (Zafri et al., 2019). To this end, since the crossing duration expresses the exposure to risks, it is of great importance to further investigate it and particularly to focus on distracted pedestrians who are considered as more vulnerable.

\section{Methodology}

\subsection{Design of the data collection process}

The current research utilizes naturalistic observations, exploiting the advantages of this methodology as mentioned in the Section of Literature Review. During the survey, only pedestrians that were using mobile phones while crossing the road were observed, at both signalized and unsignalized intersections. The number of undistracted pedestrians was also recorded, in order to quantify the frequency of distracted walking phenomenon. The collected data were relevant to:

1. site characteristics (traffic light duration for vehicles and pedestrians, road width),

2. pedestrians' demographics (gender, estimated age),

3. mobile phone task performed during crossing (e.g. holding it without using it, talking, listening to music, texting),

4. existence of other distractors (e.g. presence of companion, carriage of another object) and

5. pedestrians' crossing behavior characteristics.

Regarding pedestrian's crossing behavior, it was observed before and during the crossing. In particular, the parameters recorded were:

1. pedestrians' traffic signal (red; green) during the crossing, for signalized sites,

2. check of traffic before crossing (yes; no),

3. waiting for traffic to stop before crossing (Yes; No; No need for waiting due to no traffic),

4. look direction during crossing (straight ahead; at traffic; on the phone; at company; other),

5. pedestrian's waiting position before crossing (on the sidewalk; on the crosswalk; start crossing without waiting),

6. pedestrian's reaction to signal change from "green" to "red" indication (stop; return to initial position; acceleration),

7. conflict with a vehicle (yes; no), and

8. crossing duration (in seconds). 


\subsection{Site selection and sampling}

In order to collect an adequate and representative sample of pedestrians, the selection of a central district with high pedestrian flows and mixed land uses, was considered advisable. Furthermore, taking into account that controlled intersections are considered to be safer than uncontrolled ones, the survey included observations at both signalized and unsignalized intersections. It was also desirable to examine intersections in close proximity to each other, so as to eliminate differences in the environmental conditions. Based on these criteria, the sites were finally located in a central district of Thessaloniki, Greece, whose characteristics are presented in Tables 1 and 2.

The data were collected during the peak hours of pedestrians' traffic, from 10:00 till 14:00 in the morning and from 18:00 till 20:00 in the evening. The observer kept a safe distance from road traffic, without blocking walking trajectories. Also, the observations, which were collected

Table 1 Alexandrou Svolou Str/Ethnikis Aminis Str crossing characteristics

\begin{tabular}{|c|c|c|c|c|}
\hline Characteristics & \multicolumn{4}{|c|}{ Site } \\
\hline Location & \multicolumn{4}{|c|}{$\begin{array}{c}\text { Alexandrou Svolou Str/Ethnikis } \\
\text { Aminis Str }\end{array}$} \\
\hline $\begin{array}{l}\text { Traffic light signal for } \\
\text { pedestrians }\end{array}$ & Yes & Yes & Yes & No \\
\hline Traffic light signal for vehicles & Yes & Yes & No & No \\
\hline $\begin{array}{l}\text { Green light time for } \\
\text { pedestrians (sec) }\end{array}$ & $31-35$ & $32-33$ & 40 & - \\
\hline $\begin{array}{l}\text { Red light time for pedestrians } \\
(\mathrm{sec})\end{array}$ & $48-58$ & 57 & $30-50$ & - \\
\hline Road width (m) & 4.6 & 8.2 & 6.0 & 8.0 \\
\hline $\begin{array}{l}\text { Pedestrians using mobile phone } \\
(\%)\end{array}$ & 18.2 & 18.8 & 20.3 & 19.8 \\
\hline Total pedestrians' observations & 122 & 128 & 159 & 241 \\
\hline
\end{tabular}

Table 2 Alexandrou Svolou Str/Ippodromiou Str crossing characteristics

\begin{tabular}{lcccccc}
\hline Characteristics & \multicolumn{7}{c}{ Site } \\
\hline $\begin{array}{l}\text { Location } \\
\begin{array}{l}\text { Traffic light signal for } \\
\text { pedestrians }\end{array}\end{array}$ & No & No & No & No & No & No \\
$\begin{array}{l}\text { Traffic light signal for } \\
\text { vehicles }\end{array}$ & No & No & No & No & No & No \\
$\begin{array}{l}\text { Green light time for } \\
\text { pedestrians (sec) }\end{array}$ & - & - & - & - & - & - \\
$\begin{array}{l}\text { Red light time for } \\
\text { pedestrians (sec) }\end{array}$ & - & - & - & - & - & - \\
$\begin{array}{l}\text { Road width (m) } \\
\text { Pedestrians using } \\
\text { mobile phone (\%) }\end{array}$ & 5.7 & 3.9 & 3.9 & 6.0 & 4.0 & 4.1 \\
$\begin{array}{l}\text { Total pedestrians' } \\
\text { observations }\end{array}$ & 30 & 75 & 73 & 29 & 53 & 53 \\
\hline
\end{tabular}

under poor visibility of the observer, were excluded from the dataset. Finally, 963 valid observations of distracted pedestrians were included in the analysis (409 at signalized crossings and 554 at unsignalized crossings).

\subsection{Statistical analysis and modeling}

The data were initially analyzed in terms of descriptive statistics, employing SPSS, in order to provide some useful information about the sample of the study. Then, two statistical models were developed and interpreted, applying Bayesian regression techniques. The first model focused on unsignalized crossings and the second on signalized crossings. The pedestrian's crossing duration was determined as the dependent variable. Both models reflected the correlation of pedestrian's crossing duration with various influential factors. The analysis was carried out in $\mathrm{R}$ programming language ( $\mathrm{R}$ Core Team, 2017) using the BAS package (Clyde, 2018).

\section{Results}

\subsection{Descriptive statistics}

The sample is well distributed regarding gender, since 515 (53.48\%) females and 448 (46.52\%) males were observed. The vast majority $(69.1 \%)$ of the pedestrians were estimated to be between 18 and 39 years old, possibly due to the increased familiarity of people of that age in using mobile phones. Also, 407 (42.26\%) pedestrians were holding their mobile phones without using them. For those practically using their phones, 246 (25.5\%) were talking, 123 (12.8\%) were texting, $70(7.3 \%)$ were using headphones and $113(11.7 \%)$ were performing more than one of these tasks. The rest of the pedestrians were performing other tasks, such as fusing a camera. In regard to additional distractors while crossing, $33.3 \%$ of the pedestrians were carrying another object while crossing, while $22.1 \%$ of them were accompanied by another pedestrian. Only $5.3 \%$ of the sample had a conflict experience with a motorized vehicle.

Concerning the pedestrians' crossing behavior, the majority of the pedestrians checked the motorized traffic before crossing $(84.9 \%)$, while for those crossing a signalized crossing, 239 (58.4\%) crossed the road during the green light indication. This result is consistent with the results of a questionnaire-based survey, which took place in the same city and took into account both distracted and undistracted cases (Basbas et al., 2019). Moreover, 32.8\% of the pedestrians did not wait before crossing due to total absence of traffic. In the cases of the presence of traffic, $32.5 \%$ of the pedestrians were waiting on the sidewalk before crossing, $21.7 \%$ on the 
crosswalk and $13 \%$ of them crossed while motorized traffic was approaching them. Concerning the crossing duration, pedestrians needed on average 4.75 seconds (Standard Deviation $=\mathrm{SD}=1.355)$ to cross an unsignalized crossing and 5.46 seconds $(\mathrm{SD}=1.68)$ to cross a signalized crossing.

\subsection{Pedestrian's crossing duration at unsignalized crossings}

The conduction of the Bayesian regression analysis for pedestrians' crossing duration at unsignalized crossings led to the generation of 38,013 models. Fig. 1 illustrates the models ranked by their posterior probability, where black color indicates which features are being left out of each model.

The most plausible of the 38,013 models (highest probability model) has a posterior probability of $12.57 \%$ and it is much higher than the uniform prior probability assigned to it. This model includes an intercept, as well as the road width ("RoadWidth"), the presence of a companion during crossing ("Company"), the carrying of an object while walking ("CarrySth") and the experience of a conflict during crossing ("Conflict"). The R2 indicator is equal to $69.43 \%$, which shows that the independent variables may explain approximately $70 \%$ of the dependent variable's variance.

Fig. 2 points out the posterior distribution of the coefficients. Based on Fig. 2, it can be demonstrated that no feature has a large overlap with 0 . The vertical line corresponds to the posterior probability that the coefficient equals to 0 . The shaped curve shows the density of possible values where the coefficient is non-zero. It is worth-mentioning that the height of the line is scaled to its probability. This implies that the intercept and all the variables included in the model show no line denoting non-zero probability.
The coefficients of the variables, which were used in the most plausible model for predicting pedestrians' crossing duration at unsignalized crossings are presented in Table 3. Based on the coefficient values of Table 3 , it can be stated with $95 \%$ certainty that increasing the width of the street by $1 \mathrm{~m}$ implies a delay of 0.5 up to 0.6 seconds. In addition, when a distracted pedestrian carries another object, the crossing duration can be increased by 0 to 0.39 seconds. Respectively, there is $95 \%$ chance that the presence of accompanying persons can extend the crossing duration by 0 to 0.44 seconds. Regarding the number of conflicts with vehicles that a distracted pedestrian may experience, there is $95 \%$ possibility that an increase by one unit can result to a reduction of 0.45 up to $1.15 \mathrm{sec}-$ onds on the crossing duration. This indicates that pedestrians adapt their speed (accelerate) and possibly their trajectory in order to avoid a collision with a vehicle. The model's credibility was checked through the conduction of a 10 fold cross validation which generated a Root Mean Square Error (RMSE) equal to 0.76. Given that the dependent variable ranges from 2 to 8 seconds, the model is considered as credible.

\subsection{Pedestrian's crossing duration at signalized crossings}

The implementation of Bayesian regression analysis for pedestrian's crossing duration at signalized crossings generated 56,815 alternative models. Fig. 3 illustrates the models ranked by their posterior probability. The highest probability model has a posterior probability of $6.8 \%$ and includes an intercept, the road width ("RoadWidth"), pedestrians aged over 65 years old ("AgeOver65") and the position

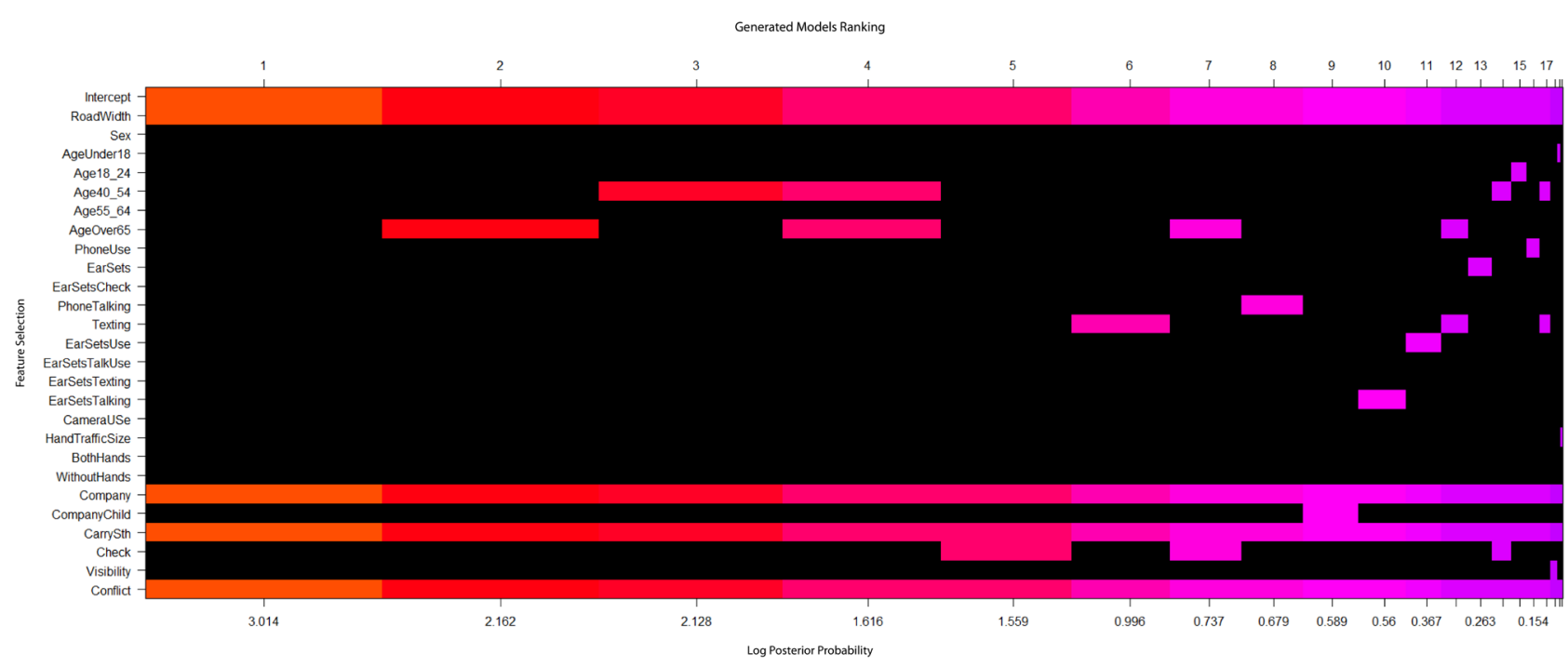

Fig. 1 Models predicting pedestrians' crossing time at unsignalized crossings ranked by their posterior probability 
Intercept

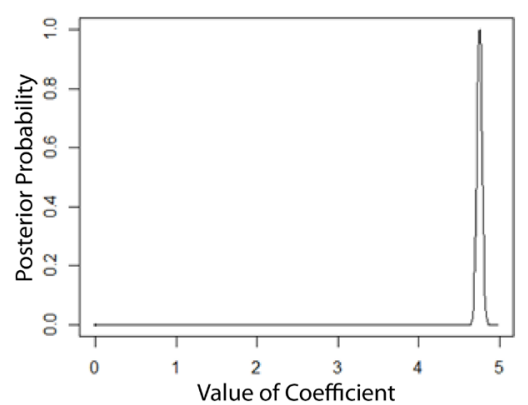

Company

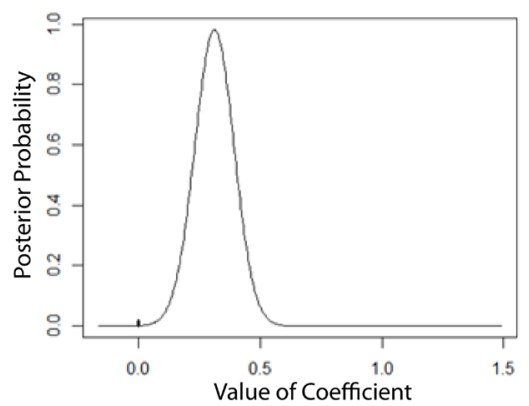

RoadWidth

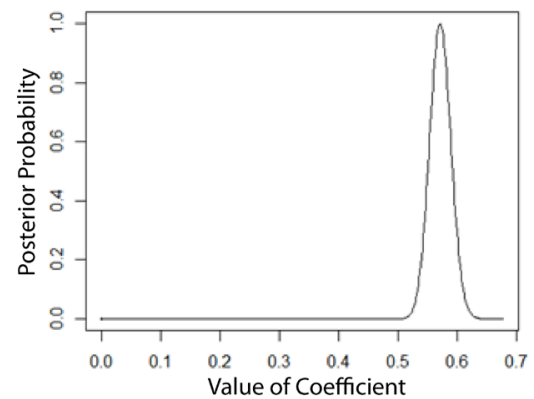

Conflict

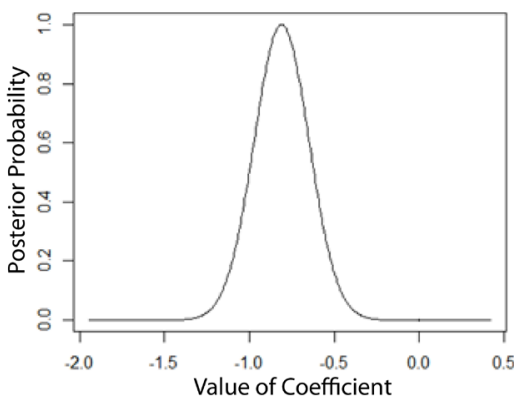

Fig. 2 Posterior distribution of the coefficients (unsignalized crossings)

Table 3 Coefficients of the features of the most plausible model predicting pedestrians crossing time at unsignalized crossings

\begin{tabular}{lcccc}
\hline Variable & post mean & post SD & $2.5 \%$ & $97.5 \%$ \\
\hline Intercept & 4.75 & 0.03 & 4.69 & 4.81 \\
RoadWidth & 0.56 & 0.05 & 0.53 & 0.60 \\
CarrySth & 0.25 & 0.11 & 0 & 0.39 \\
Company & 0.29 & 0.11 & 0 & 0.44 \\
Conflict & -0.79 & 0.19 & -1.15 & -0.45 \\
\hline
\end{tabular}

of the pedestrian before crossing ("StandingCrossing"). The posterior probability of the model is much higher than the uniform prior probability. The value of R2 highlights that the independent variables included in the model can explain $12 \%$ of the dependent variables' variance.

In Fig. 4, the posterior distribution of the coefficients for the signalized crossings is illustrated in accordance with the process followed for the modeling of crossing duration

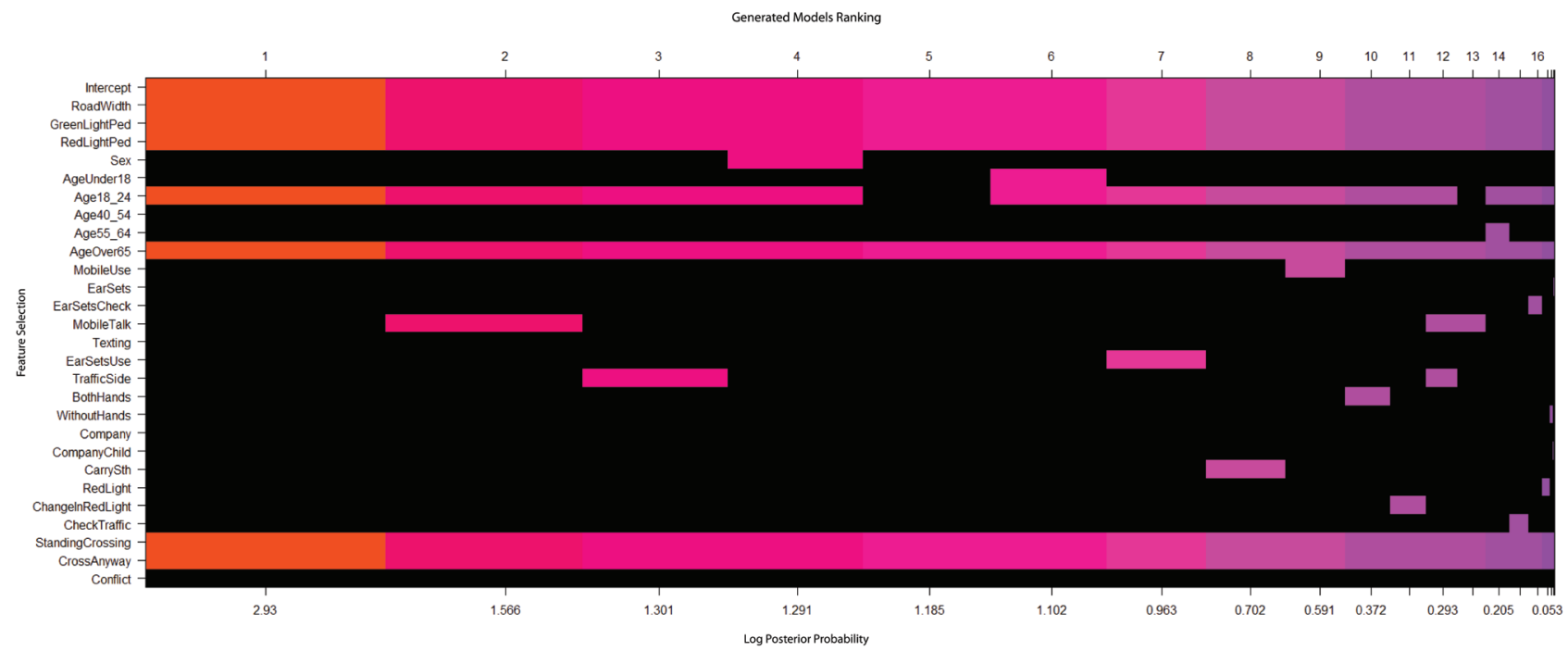

Fig. 3 Models predicting pedestrians' crossing time at signalized crossings ranked by their posterior probability 
Intercept

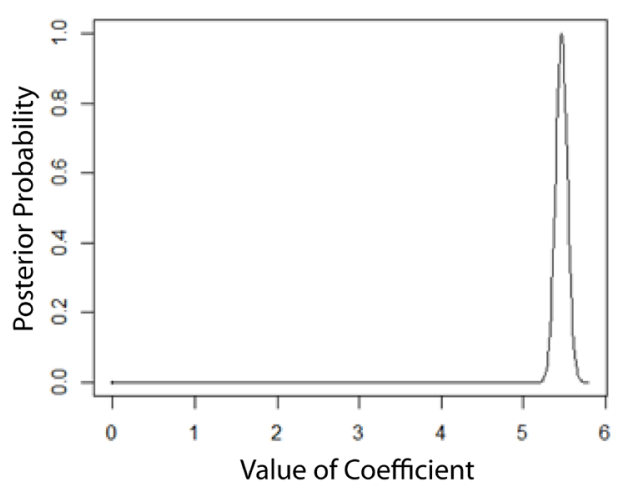

RoadWidth

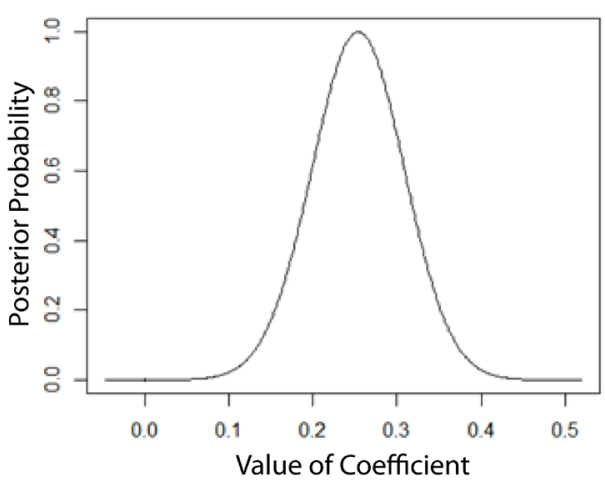

StandingCrossing

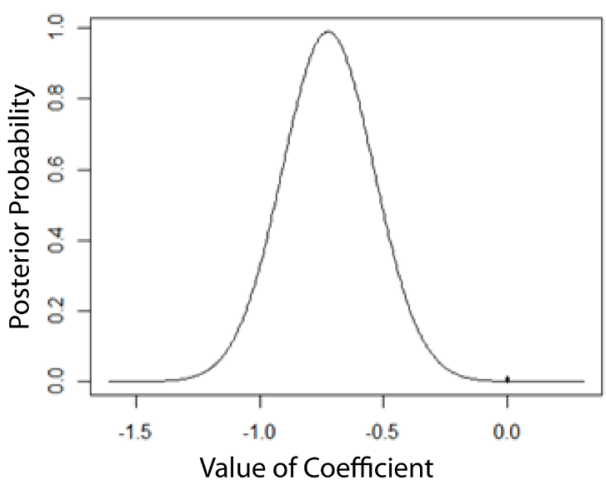

AgeOver65

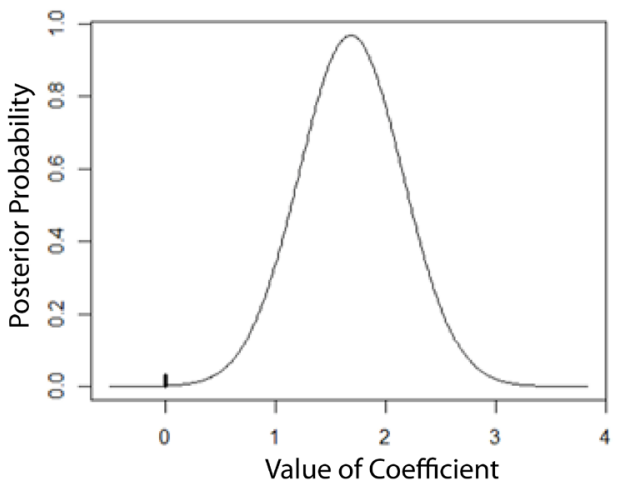

Fig. 4 Posterior distribution of the coefficients (signalized crossings)

at unsignalized intersections. Based on Fig. 4, it can be demonstrated that no feature that has a large overlap with 0 , such as in the model for unsignalized crossings.

The coefficient values of features used in the most plausible model for predicting pedestrians' crossing duration at signalized crossings are presented in Table 4. Based on these values, it can be concluded with $95 \%$ certainty that increasing the width of the street by $1 \mathrm{~m}$, crossing duration can be delayed by 0.19 to 0.42 seconds. Furthermore, when a pedestrian waits on the crosswalk before crossing, there is $95 \%$ chance that the crossing duration can be reduced by 0.3 to 1.33 seconds. This indicates a more offensive behavior of those waiting on the crosswalk compared with those

Table 4 Coefficients of the features of the most plausible model predicting pedestrians crossing time at signalized crossings

\begin{tabular}{lcccc}
\hline Variable & post mean & post SD & $2.5 \%$ & $97.5 \%$ \\
\hline Intercept & 5.46 & 0.08 & 5.31 & 5.62 \\
RoadWidth & 0.31 & 0.06 & 0.19 & 0.42 \\
StandingCrossing & -0.73 & 0.3 & -1.33 & -0.3 \\
AgeOver65 & 1.57 & 0.83 & 0 & 2.69 \\
\hline
\end{tabular}

waiting on the sidewalk. Moreover, there is $95 \%$ possibility that the crossing duration of the elderly can be increased by 0 to 2.69 seconds in comparison with younger pedestrians.

The model's credibility was checked through the conduction of a 10 fold cross validation which generated a Root Mean Square Error (RMSE) equal to 1.5. Given that the dependent variable ranges from 3 to 12 seconds, this model is also considered to be credible.

\section{Discussion}

The present paper deals with distracted walking, which is currently a usual phenomenon, due to the increased use of portable electronic devices and mainly mobile phones. This fact is also validated by the results of this paper, since approximately a $15-20 \%$ of pedestrians were observed to cross the road while holding or using a mobile phone. The most common type of mobile phone usage, as recorded in this survey, is talking without hands-free device. However, texting while crossing the road is also found to be very common, which has also been highlighted by the findings of previous surveys. 
The analysis focuses on crossing duration, which is an essential variable, because it expresses the exposure time of the pedestrian at collisions with vehicles. Except for the road width, different factors were found to be influential between signalized and unsignalized crossings. The results from the analysis of the unsignalized crossings indicate that distracted pedestrians' exposure time is increased when carrying an object or when crossing with a companion. Also, the experience of a conflict with a vehicle during crossing modifies the crossing behavior and more specifically it influences the pedestrians to accelerate. Regarding signalized crossings, the age found to be one of the most influential factors, indicating that the exposure duration of the elderly at risks is much higher compared to younger pedestrians. Also, pedestrians, who wait on the crosswalk, show a more offensive attitude compared to those waiting on the sidewalk and this is reflected in the crossing duration.

The current findings can complement the existing literature on the subject of distracted walking emphasizing on mobile phone use while crossing. They can also provide

\section{References}

Agostini, V., Lo Fermo, F., Massazza, G., Knaflitz, M. (2015) "Does texting while walking really affect gait in young adults?", Journal of NeuroEngineering and Rehabilitation, 12(1), Article number: 86. https://doi.org/10.1186/s12984-015-0079-4

Barin, E. N., McLaughlin, C. M., Farag, M. W., Jensen, A. R., Upperman, J. S., Arbogast, H. (2018) "Heads Up, Phones Down: A Pedestrian Safety Intervention on Distracted Crosswalk Behavior", Journal of Community Health, 43(4), pp. 810-815. https://doi.org/10.1007/s10900-018-0488-y

Bansal, A., Goyal, T., Sharma, U. (2019) "Modelling the Pedestrian Speed at Signalised Intersection Crosswalks for Heterogeneous Traffic Conditions", Promet - Traffic \& Transportation, 31(6), pp. 681-692.

https://doi.org/10.7307/ptt.v31i6.3299

Barton, B. K., Kologi, S. M., Siron, A. (2016) "Distracted pedestrians in crosswalks: An application of the Theory of Planned Behavior", Transportation Research Part F: Traffic Psychology and Behaviour, 37, pp. 129-137.

https://doi.org/10.1016/j.trf.2015.12.012

Basbas, S., Nikiforiadis, A., Sarafianou, E., Kolonas, N. (2019) "Behavior and Perceptions of University Students at Pedestrian Crossings", In: Nathanail, E., Karakikes, I. (eds.) Data Analytics: Paving the Way to Sustainable Urban Mobility, Springer, Cham, Switzerland, pp. $280-287$.

https://doi.org/10.1007/978-3-030-02305-8_34

Chen, P.-L., Saleh, W., Pai, C.-W. (2017) "Texting and walking: a controlled field study of crossing behaviours and inattentional blindness in Taiwan", Behaviour \& Information Technology, 36(4), pp. $435-445$.

https://doi.org/10.1080/0144929X.2016.1240234 further information on the factors that have a negative or positive impact on the duration of crossing a road, indicating which of them can pose higher risk to the pedestrians' road safety. These factors can be exploited in the building of prediction models and the simulation of distracted walking caused by mobile phone use, which can contribute to the development of more reliable and safe automated vehicles in the near future. Last but not least, it can assist efficiently engineers and policy makers, who attempt to apply appropriate and effective road safety measures, policies, as well as awareness campaigns towards the mitigation of distracted walking. Future research would include the conduction of a similar research aiming at identifying whether there are any differences between the exposure time of distracted and undistracted pedestrians. In this framework, the identification of the overall potential factors that affect crossing duration of pedestrians, distracted by mobile phone use, would be complemented and the construction of more precise and holistic models of distracted walking would be facilitated.

Chen, P.-L., Pai, C.-W. (2018) "Pedestrian smartphone overuse and inattentional blindness: an observational study in Taipei, Taiwan", BMC Public Health, 18(1), Article number: 1342. https://doi.org/10.1186/s12889-018-6163-5

Chen, P.-L., Saleh, W., Pai, C.-W. (2018) "Pokemon gaming causes pedestrians to run a red light: An observational study of crossing behaviours at a signalised intersection in Taipei City", Transportation Research Part F: Traffic Psychology and Behaviour, 55, pp. 380-388. https://doi.org/10.1016/j.trf.2018.03.011

Clyde, M. (2018) "BAS: Bayesian Variable Selection and Model Averaging using Bayesian Adaptive Sampling, (R package version 1.5.3)", [computer program] Available at: https://cran.r-project.org/web/ packages/BAS/BAS.pdf [Accessed: 15 September 2019]

Feld, J. A., Plummer, P. (2019) "Visual scanning behavior during distracted walking in healthy young adults", Gait \& Posture, 67, pp. 219-223. https://doi.org/10.1016/j.gaitpost.2018.10.017

Forde, A., Daniel, J. (2020) "Pedestrian walking speed at un-signalized midblock crosswalk and its impact on urban street segment performance", Journal of Traffic and Transportation Engineering (English Edition). https://doi.org/10.1016/j.jtte.2019.03.007

Galanis, A., Botzoris, G., Eliou, N. (2017) "Pedestrian road safety in relation to urban road type and traffic flow", Transportation Research Procedia, 24, pp. 220-227. https://oi.org/10.1016/j.trpro.2017.05.111

Gary, C. S., Lakhiani, C., DeFazio, M. V., Masden, D. L., Song, D. H. (2018) "Caution with Use: Smartphone-Related Distracted Behaviors and Implications for Pedestrian Trauma", Plastic and Reconstructive Surgery, 142(3), p. 428e. https://doi.org/10.1097/PRS.0000000000004642 
Haga, S., Sano, A., Sekine, Y., Sato, H., Yamaguchi, S., Masuda, K. (2015) "Effects of using a Smart Phone on Pedestrians' Attention and Walking", Procedia Manufacturing, 3, pp. 2574-2580. https://doi.org/10.1016/j.promfg.2015.07.564

Hassouna, F. M. A. (2020) "Evaluation of Pedestrian Walking Speed Change Patterns at Crosswalks in Palestine", The Open Transportation Journal, 14, pp. 44-49. https://doi.org/10.2174/1874447802014010044

Hatfield, J., Murphy, S. (2007) "The effects of mobile phone use on pedestrian crossing behaviour at signalised and unsignalised intersections", Accident Analysis and Prevention, 39(1), pp. 197-205. https://doi.org/10.1016/j.aap.2006.07.001

Hyman, I. E., Boss, S. M., Wise, B. M., McKenzie, K. E., Caggiano, J. M. (2010) "Did you see the unicycling clown? Inattentional blindness while walking and talking on a cell phone", Applied Cognitive Psychology, 24(5), pp. 597-607. https://doi.org/10.1002/acp.1638

Jiang, K., Ling, F., Feng, Z., Ma, C., Kumfer, W., Shao, C., Wang, K. (2018) "Effects of mobile phone distraction on pedestrians' crossing behavior and visual attention allocation at a signalized intersection: An outdoor experimental study", Accident Analysis and Prevention, 115, pp. 170-177. https://doi.org/10.1016/j.aap.2018.03.019

Kadali, B. R., Vedagiri, P. (2019) "Evaluation of pedestrian crossing speed change patterns at unprotected mid-block crosswalks in India", Journal of Traffic and Transportation Engineering (English Edition). https://doi.org/10.1016/j.jtte.2018.10.010

Krasovsky, T., Weiss, P. L., Kizony, R. (2017) "A narrative review of texting as a visually-dependent cognitive-motor secondary task during locomotion", Gait \& Posture, 52, pp. 354-362. https://doi.org/10.1016/j.gaitpost.2016.12.027

Lamberg, E. M., Muratori, L. M. (2012) "Cell phones change the way we walk", Gait \& Posture, 35(4), pp. 688-690. https://doi.org/10.1016/j.gaitpost.2011.12.005

Lambrianidou, P., Basbas, S., Politis, I. (2013) "Can pedestrians' crossing countdown signal timers promote green and safe mobility?", Sustainable Cities and Society, 6, pp. 33-39. https://doi.org/10.1016/j.scs.2012.07.005

Lennon, A., Williamson, A., King, M., Lewis, I., Haque, M. (2016) "Distraction and Attitudes Towards Safe Pedestrian Behaviour", [pdf] Austroads Ltd., Sidney, Australia, Rep. AP-R510-16. Available at: http:/transit.gencat.cat/web/.content/OSV/documents/ ambit_local/OSV_AP_R510_16.pdf [Accessed: 15 February 2020]

Licence, S., Smith, R., McGuigan, M., Earnest, C. P. (2015) "Gait Pattern Alterations during Walking, Texting and Walking and Texting during Cognitively Distractive Tasks while Negotiating Common Pedestrian Obstacles", Plos One, 10(7), Article number: e0133281. https://doi.org/10.1371/journal.pone.0133281

Lin, M.-I. B., Huang, Y.-P. (2017) "The impact of walking while using a smartphone on pedestrians' awareness of roadside events", Accident Analysis and Prevention, 101, pp. 87-96. https://doi.org/10.1016/j.aap.2017.02.005

Magnani, R. M., Lehnen, G. C., Rodrigues, F. B., de Sá e Souza, G. S., de Oliveira Andrade, A., Vieira, M. F. (2017) "Local dynamic stability and gait variability during attentional tasks in young adults", Gait \& Posture, 55, pp. 105-108.

https://doi.org/10.1016/j.gaitpost.2017.04.019
Mohd Syazwan, S., Baba, M. D., Nur Zarifah, H., Aqbal Hafeez, A., Noor Faradila, P. (2017) "Prevalence of distracted pedestrians while crossing: a study of Malaysia's situation", MATEC Web of Conferences, 90, Article number: 01031. https://doi.org/10.1051/matecconf/20179001031

Muley, D., Kharbeche, M., Alhajyaseen, W., Al-Salem, M. (2017) "Pedestrians' Crossing Behavior at Marked Crosswalks on Channelized Right-Turn Lanes at Intersections", Procedia Computer Science, 109, pp. 233-240. https://doi.org/10.1016/j.procs.2017.05.339

Mwakalonge, J., Siuhi, S., White, J. (2015) "Distracted walking: Examining the extent to pedestrian safety problems", Journal of Traffic and Transportation Engineering (English Edition), 2(5), pp. $327-337$. https://doi.org/10.1016/j.jtte.2015.08.004

Neider, M. B., McCarley, J. S., Crowell, J. A., Kaczmarski, H., Kramer, A. F. (2010) "Pedestrians, vehicles, and cell phones", Accident Analysis and Prevention, 42(2), pp. 589-594. https://doi.org/10.1016/j.aap.2009.10.004

Ortiz, N. C., Ramnarayan, M., Mizenko, K. (2017) "Distraction and road user behavior: An observational pilot study across intersections in Washington, D.C.", Journal of Transport \& Health, 7(Part A), pp. 13-22. https://doi.org/10.1016/j.jth.2017.05.362

Paschalidis, E., Politis, I., Basbas, S., Lambrianidou, P. (2016) "Pedestrian compliance and cross walking speed adaptation due to countdown timer installations: A self-report study", Transportation Research Part F: Traffic Psychology and Behaviour, 42(Part 3), pp. 456-467. https://doi.org/10.1016/j.trf.2015.07.010

Pešić, D., Antić, B., Glavić, D., Milenković, M. (2016) "The effects of mobile phone use on pedestrian crossing behavior at unsignalized intersections - Models for predicting unsafe pedestrians behaviour", Safety Science, 82 , pp. 1-8. https://doi.org/10.1016/j.ssci.2015.08.016

Pizzamiglio, S., Naeem, U., Réhman, S. U., Saeed Sharif, M., Abdalla, H., Turner, D. L. (2017) "A Multimodal Approach to Measure the Distraction Levels of Pedestrians using Mobile Sensing", Procedia Computer Science, 113, pp. 89-96. https://doi.org/10.1016/j.procs.2017.08.297

Plummer, P., Apple, S., Dowd, C., Keith, E. (2015) "Texting and walking: Effect of environmental setting and task prioritization on dualtask interference in healthy young adults", Gait \& Posture, 41(1), pp. 46-51. https://doi.org/10.1016/j.gaitpost.2014.08.007

R Core Team (2017) "R: A language and environment for statistical computing", R Foundation for Statistical Computing, Vienna, Austria. [online] Available at: https://www.R-project.org/ [Accessed: 15 February 2020]

Ropaka, M., Nikolaou, D., Mavromatis, S., Yannis, G. (2020) "Investigation of Traffic and Safety Behavior of Pedestrians Texting or Web-Surfing", presented at Transportation Research Board (TRB) $99^{\text {th }}$ Annual Meeting, TRBAM 2020, Washington, D.C., USA, Jan., 12-16, 2020. 
Russo, B. J., James, E., Aguilar, C. Y., Smaglik, E. J. (2018) "Pedestrian Behavior at Signalized Intersection Crosswalks: Observational Study of Factors Associated with Distracted Walking, Pedestrian Violations, and Walking Speed", Transportation Research Record: Journal of the Transportation Research Board, 2672(35), pp. 1-12. https://doi.org/10.1177/0361198118759949

Shaaban, K. (2019) "Analysis of Pedestrian Crossing Speeds at Signalized Intersections in Qatar", Arabian Journal for Science and Engineering, 44(5), pp. 4467-4476. https://oi.org/10.1007/s13369-018-3460-3

Scopatz, R. A., Zhou, Y. (2016) "Effect of Electronic Device Use on Pedestrian Safety: A literature Review", [pdf] National Highway Traffic Safety Administration (NHTSA), Washington, D.C., USA, Rep. DOT HS 812 256. Available at: https://www.nhtsa.gov/sites/ nhtsa.dot.gov/files/812256-effectelectronicdeviceusepedestriansa fety.pdf [Accessed: 15 February 2020]

Sobhani, A., Farooq, B. (2018) "Impact of smartphone distraction on pedestrians' crossing behaviour: An application of head-mounted immersive virtual reality", Transportation Research Part F: Traffic Psychology and Behaviour, 58, pp. 228-241.

https://doi.org/10.1016/j.trf.2018.06.020
Tapiro, H., Oron-Gilad, T., Parmet, Y. (2016) "Cell phone conversations and child pedestrian's crossing behavior; a simulator study", Safety Science, 89 , pp. 36-44. https://doi.org/10.1016/j.ssci.2016.05.013

Wells, H. L., McClure, L. A., Porter, B. E., Schwebel, D. C. (2018) "Distracted Pedestrian Behavior on two Urban College Campuses", Journal of Community Health, 43(1), pp. 96-102. https://doi.org/10.1007/s10900-017-0392-X

World Health Organization, NHTSA (2011) "Mobile Phone Use: A Growing Problem of Driver Distraction", [pdf] World Health Organization, Geneva, Switzerland. Available at: https://apps. who.int/iris/bitstream/handle/10665/44494/9789241500890_eng. pdf? sequence=1\&isAllowed=y [Accessed: 15 February 2020]

Zafri, N. M., Rony, A. I., Adri, N. (2019) "Analysis of Pedestrian Crossing Speed and Waiting Time at Intersections in Dhaka", Infrastructures, 4(3), Article number: 39. https://doi.org/10.3390/infrastructures4030039 